\title{
Experimental Evaluation of the Cardiac Rhythm Originating in Myocardial Sleeves of Pulmonary Veins Using a Monophasic Action Potential
}

\author{
O. KITTNAR ${ }^{1}$, S.-G. YANG ${ }^{1}$, M. MLČEK ${ }^{1}$ \\ ${ }^{1}$ Institute of Physiology, First Faculty of Medicine, Charles University, Prague, Czech Republic
}

Received April 26, 2013

Accepted June 25, 2013

\section{Summary}

Spontaneous depolarization similar to that from the sinus node was documented from the myocardial sleeves of pulmonary veins (PV) after isolation procedures. It was then hypothesized that sinus node-like tissue is present in the PVs of humans. Based on a number of features, the myocardium of myocardial sleeves (MS) is highly arrhythmogenic. Membrane potentials originating from MS are invariably recordable at the PVs ostia in patients with atrial fibrillation (AF) and delayed conduction around the PVs ostia may play a role in re-entry process responsible for the initiation and maintenance of AF. Diagnostic and therapeutic evidence of premature atrial beats induced in MS of PVs and resulting in launch of $A F$ was detected by $3 D$ electroanatomic method of monophasic action potential (MAP). MAP recording plays an important role in a direct view of human myocardial electrophysiology under both physiological and pathological conditions. Its crucial importance lies in the fact that it enables the study of the action potential of myocardial cell in vivo and, therefore, the study of the dynamic relation of this potential with all the organism variables. The knowledge of pathological MAPs from PV myocardial sleeves can help us to confirm a diagnosis when finding the similar action potential morphology. MAP can be also used to evaluate the therapeutic efficiency of vagal nerves suppression, radiofrequency ablation or other treatment procedures in PVs myocardial sleeves as well as for posttreatment following up.

\section{Key words}

Myocardial sleeves - Pulmonary veins • Monophasic action potential • Atrial fibrillation

\section{Corresponding author}

Otomar Kittnar, Institute of Physiology, First Faculty of Medicine, Charles University in Prague, Albertov 5, 12800 Prague 2, Czech Republic. E-mail: otomar.kittnar@staff.cuni.cz

\section{Introduction}

From all known arrhythmias atrial fibrillation is the most common one (Go et al. 2001). It is characterized by turbulence-like electrical activation of atrial tissue, leading to desynchronization of contraction (Zemlin et al. 2009) and most commonly, it is triggered by rapid bursts of electrical impulses originating in the extensions of the left atrial myocardium over the pulmonary veins (PV), so-called myocardial sleeves of pulmonary veins. Membrane potentials from myocardial sleeves are invariably recordable at the PV ostia in patients with atrial fibrillation (AF) and delayed conduction around the PV ostia may play a role in re-entry process responsible for the initiation and maintenance of AF (Hertervig et al. 2008).

Monophasic action potential (MAP) recordings of myocardial cells offer highly accurate information on the action potential duration and its configuration, including early after-depolarizations as well as relative changes in transmembrane diastolic and systolic potential changes and thus probably reflect the transmembrane voltage of cells (Franz 1991, Yang and Kittnar 2010). Therefore, the MAP analysis can play an important role in studying the significance of myocardial sleeves of PV in triggering and sustaining AF. 


\section{History}

Nevertheless there was a long and complicated way to reach this understanding of the origin and mechanisms of atrial fibrillation (AF). At the beginning of the 20th century chest palpitations and an irregular and rapid pulse was more definitively diagnosed as atrial fibrillation thanks to the advent of the electrocardiogram. The discovery of a nature of this most common arrhythmia opened for many decades an endless dispute about its underlying mechanism ( $\mathrm{He}$ et al. 2012). Particularly two theories dominated. Focal theory of AF was based on findings that substances, such as aconitine (Scherf 1947) or acetylcholine applied to the atrial appendage or to the area of the sinoatrial or atrioventricular nodes, (Scherf et al. 1950) could induce tachycardia or AF. As either isolation or local cooling of the appendage lead to suppression of tachyarrhythmias the resulting conclusion was that $\mathrm{AF}$ was focal. Multiple wavelet theory was derived from experiments that brought an evidence of reentrant circuits orbiting in atrial myocardium during $\mathrm{AF}$ triggered by induced atrial extrasystoles and simultaneous stimulation of vagal nerve (Moe 1962).

Common for both theories is involvement of autonomic nervous system confirmed by a phenomenon that stimulation of the vagosympathetic trunks shortens heterogeneously a refractory period in atria, in other words it causes dispersion of refractoriness (Hoff and Geddes 1955). Just this phenomenon allows premature beats to induce and sustain AF. However innervation of the heart is a complex of extrinsic innervation from central nervous system and intrinsic system composed from hundreds of neurons in the ganglia plexi on the heart itself (Armour et al. 1997, Pauza et al. 2000) and majority of these plexi is situated at the PVs close to their entrance into left atrium.

Anatomical structure called myocardial sleeves (MS) of PV, was first described in 1836 in dissertation thesis "De arteriarum et venarum structura" of Ferdinand Raeuschel, student of the Faculty of Medicine in then Prussian Breslau (now Polish Wroclaw) at the Department of Physiology under the leadership of Jan E. Purkinje (Steiner 2005). A detailed gross anatomical description of MS was then published 130 years later by Nathan and Eliakim (1966). Meantime, in 1876, Brunton and Fayrer described spontaneous, independent pulsations of the PVs (Fye 1989) but only in the late 1990s, after the pioneering electrophysiological studies of
Jaïs and Haïssaguerre (Jaïs et al. 1996, Haïssaguerre et al. 1998), the crucial role of PVs in the initiation of AF was generally accepted and subsequently the sleeves became center of interest for many specialists in morphology, physiology and cardiology.

\section{The role of myocardial sleeves in atrial arrhythmogenesis}

First important information can be found in embryologic consequences of the ontogenetic development of the conduction system of the heart. The location of the precursors of the conduction system is determined during embryological development of the heart by the looping process of the cardiac tube. Specialized conduction tissue that is derived from the tube and is destined to have pacemaker activity has been shown to be located within the myocardial sleeves of the PVs (Perez-Lugones et al. 2003). Spontaneous depolarization similar to that from the sinus node was documented from the pulmonary veins after isolation procedures. It was then hypothesized that sinus node-like tissue is present in the pulmonary veins of humans. The presence of such tissue would provide an explanation for the observation that electrical activity within the PVs is commonly observed after electrical disconnection of the PV musculature from the atrium and also would provide an understanding for why the PVs are commonly identified as the location of pacemaker electrical activity that triggers the development of AF.

Second important information is derived from the anatomic position of MS and their histological characteristic. MS are developed in $68-100 \%$ of PVs and their length is $4-48 \mathrm{~mm}$, mostly $10-13 \mathrm{~mm}$. Their mean thickness is $1.1 \mathrm{~mm}$ but may be up to $5 \mathrm{~mm}$; they are thickest at the veno-atrial junction, thinning out peripherally. The sleeves are more extensive around the superior PVs. Their peripheral margin is irregular, usually not circumferential, with multiple, small fingerlike projections (Tan et al. 2006). The myocardial bundles of the MS are arranged circularly, longitudinally and obliquely. There is significant interindividual and intraindividual variability in configuration of the sleeves.

Based on a number of features, the PVs are highly arrhythmogenic (Chen et al. 2006). The PV-left atrial (LA) junction has discontinuous myocardial fibers separated by fibrotic tissues. The PV muscle sleeve is highly anisotropic. The vein of Marshall in humans has 
multiple small muscle bundles separated by fibrosis and fat. Insulated muscle fibers can promote automaticity and thus trigger reentrant activity. The existence of interstitial cells identical with interstitial Cajal-like cells was proved in human MS (Gherghiceanu et al. 2008). These cells appear located among the myocardial cells and particularly at the border between the myocardial sleeve and pulmonary vein wall and they have long and thin cytoplasmic processes, which form an interstitial cellular network connecting cardiomyocytes, nerves, blood vessels and interstitial immune cells. According to Gherghiceanu et al. (2008) they could be a key factor in cardiac response to the mechanical stretch induced by the blood flow in the pulmonary veins under normal and/or pathological conditions. The spatial distribution of pressure sensitive autonomic nerves receptors are probably related to sites of $\mathrm{AF}$ origin (Olsson 2001). The MS also contain periodic large pale cells that correspond morphologically to Purkinje cells. These special cells are supposed to be the sources of focal discharge. Chronic sustained AF is resulting in structural remodeling (i.e. inhomogeneous increase of extracellular collagen matrix) that involves both atrial myocardium and MS. Reduced coupling of the myocytes in PVs due to histological changes may provide an additional mechanism facilitating tachyarrhythmias.

Thirdly, electrophysiological characteristics are crucial for understanding the roles of the MS. Electrocardiograms recorded in the PVs reveal double and often fractionated characteristics (de Bakker et al. 2002). Double potentials reflect remote atrial activation together with activation of the MS. Fractionation of the electrocardiograms can be attributed to the anisotropic characteristics of the atrial myocardium within the PVs. This anisotropic structure, together with the observation that the refractory period is shorter at the distal end of the PVs compared to the proximal end, may explain the frequent occurrence of conduction block in the pulmonary veins. The cycle length of bursts of ectopic beats arising in the PV of man is often irregular, supporting a focal mechanism of the ectopic beats. The anisotropic characteristics of the PVs may enhance the ability of a focus to activate the PVs and the atrium and lead to AF. De Bakker et al. (2002) identified also zones of conduction delay in all PVs. Zones of slow conduction correlated with different fiber orientation observed on histology. These changes could facilitate reentry.

\section{Pathophysiology of atrial fibrillation from the MS}

We have already concluded that MS could affect cardiac rhythm and cause atrial fibrillation. Histological analysis revealed extension of atrial myocardium into $89 \%$ of all PVs. Atrial tissue on the PVs of patients with $\mathrm{AF}$ was reported to have shorter refractory periods than in control patients or in other parts of atria in patients with AF (Hertervig et al. 2008). The refractory period is shorter in atrial tissue in the distal PVs than at the PV-left atrium junction. Decreasing conduction in PVs is more frequent in AF patients than in controls, and AF is more readily induced during pacing in the PVs than in the LA. This heterogeneity of conduction may promote reentry and form a substrate for sustained AF.

The onset and maintenance of a tachyarrhythmia require both an initiating event and an anatomical substrate. With respect to AF, the situation is often complex, and available data support a 'focal' mechanism involving automaticity or multiple reentrant wavelets (Nitta et al. 2004). Focal theory suggests that the arrhythmia persists only in isolated regions of atrial myocardium. This theory received minimal attention until a focal source for AF was observed in humans and ablation of this source stopped AF. The duration of episodes of AF correlated with both a decrease in atrial refractoriness and shortening of the AF cycle length, attesting to the importance of electrical remodeling in the maintenance of AF. Multiple-wavelet hypothesis as the mechanism of reentrant AF was advanced by finding of wavefronts propagating through the atria and resulting in self-perpetuating 'daughter wavelets' (Nitta et al. 2004). In this model, the number of wavelets at any time depends on the refractory period, mass, and conduction velocity in different parts of the atria. A large atrial mass with a short refractory period and delayed conduction increases the number of wavelets, favoring sustained AF. Atrial refractoriness is increasing with age in both men and women, but concurrent age-related fibrosis lengthens effective intra-atrial conduction pathways what in combination with the shorter wavelengths of reentrant impulses increases the likelihood of developing AF. Also amyloidosis and particularly scarring of the myocardial sleeves of the PVs appear to be common in the elderly population as an arrhythmogenic substrate for $\mathrm{AF}$ (Steiner et al. 2006). In patients with AF, typical MS potentials with marked conduction time delay were almost invariably recordable at the PV ostium, but in 


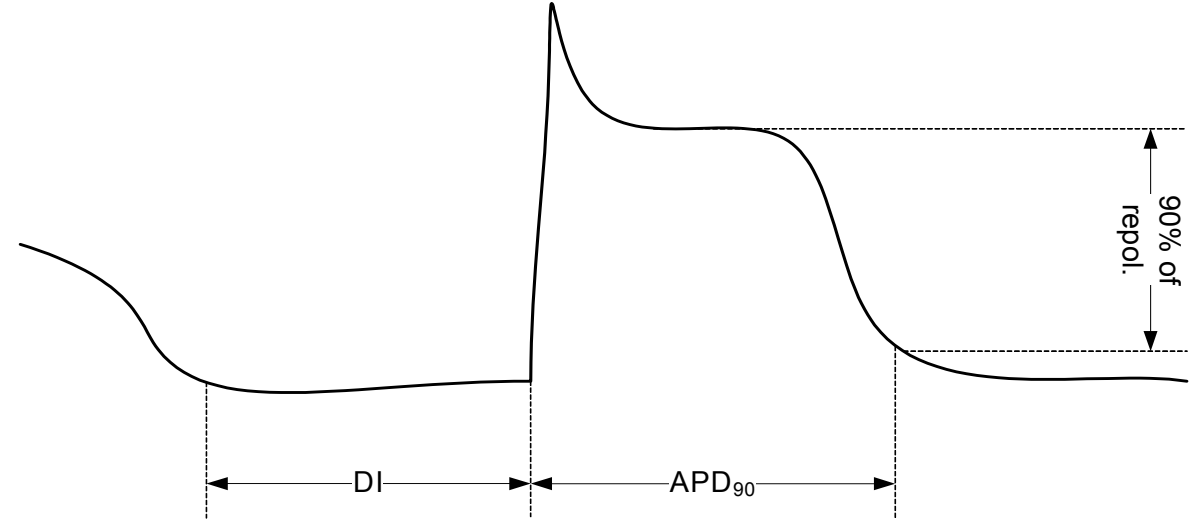

Fig. 1. Measurement of duration of action potential (ADP) and diastolic interval (DI) from MAP recording. patients without a history of $\mathrm{AF}$, merely simple, narrow potentials were found. These findings support the involvement of conduction delay and re-entrant activities around the PV ostia in the genesis and/or perpetuation of AF.

\section{Introduction of monophasic action potential (MAP)}

Electrocardiography (ECG) is the best-known and the most popular procedure of recording of the electrical activity of myocardium. However, ECG detects just body surface projection of the electrical heart field and cannot reveal heart's local information such as actual cellular depolarization and repolarization process of myocardial tissue. The standard surface electrocardiograms as well as the intracavitary electrocardiographic recordings are not able to provide more precise and more locally oriented information as they represent just the summation of an electric activity of many myocardial cells from relatively big regions of the heart (Yang and Kittnar 2010). For more detailed analysis of electrical events at a cellular level only two methods are available: the cellular impalement technique and the monophasic action potential (MAP) method. Thus, MAP recording plays an important role in a more direct view of human myocardial electrophysiology under both physiological and pathological conditions. Its crucial importance lies in the fact that it enables the study of the action potential of myocardial cell in vivo and, therefore, the study of the dynamic relation of this potential with all the organism variables (Leirner and Cestari 1999).

The procedure of MAP measuring can be simply realized using the Seldinger technique, when MAP catheter is inserted through femoral vein into the right ventricle or through femoral artery to the left ventricle.
The tip electrode has to be nearly perpendicular to endocardium what allows flexions back and forth with each cardiac contraction-relaxation cycle. The MAP catheter lead is connected to an electrophysiologic recording system and the signal can be analyzed by automated computer system (Tsalikakis et al. 2003) what allows to calculate 2D (two-dimensionally) or even $3 \mathrm{D}$ (three-dimensionally) endocardial mapping by MAPs.

\section{MAP recordings in explanation of a role of PVs in initiation of AF}

MAP technique is thus suitable study mechanism that could explain why single premature atrial complexes from the PVs may in human initiate AF. The principle consists in possibility to measure changes in the action potential duration (APD) caused by changes in the diastolic interval (DI) from the prior beat (Fig. 1). Narayan et al. (2008) studied MAPs in superior PVs of 27 patients with paroxysmal and persistent AF. They investigated relation between DI and APD and showed that in patients with paroxysmal AF, the APD shortened to a greater extent than the DI while in patients with permanent AF the relative APD lengthened. Moreover the activation time prolongation was greater in persistent than in paroxysmal AF. These differences can reflect either electrical or structural remodeling of atria what was also proved by animal experiments (Alessie et al. 2002). The crucial parameter seems to be so called restitution slope (the slope of the linear portion of the APD on DI dependence in stimulated premature atrial beats - Fig. 2). The steeper slope corresponds to the increased ability of premature beats to cause exaggerated APD oscillations that may lead to wave break and AF. It could be hypothesized that these changes in the APD near PVs may explain both the initiation and propagation of $\mathrm{AF}$. 


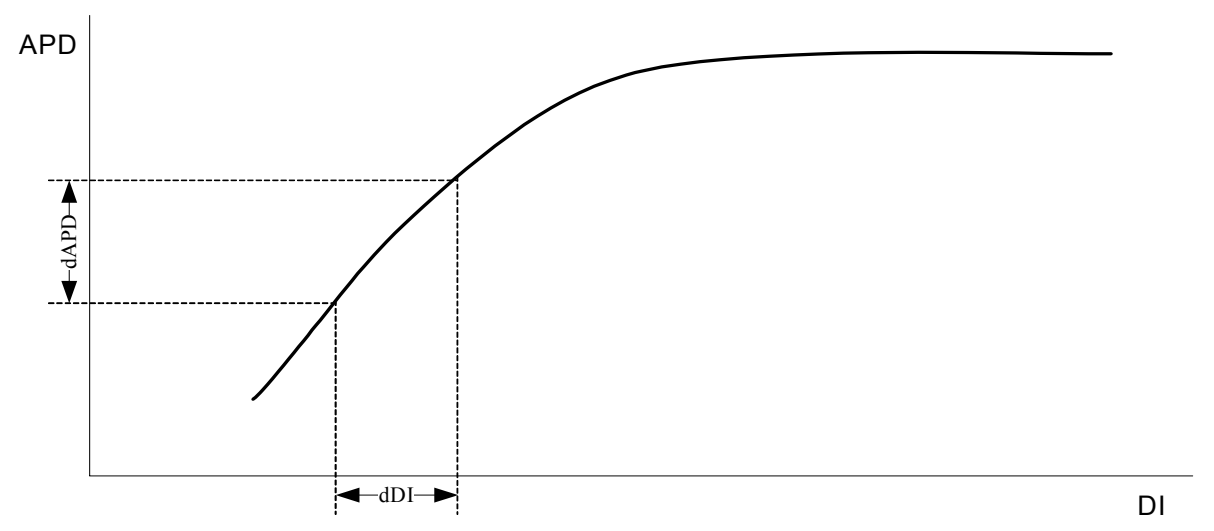

Fig. 2. Calculation of restitution slope (RS). RS = dADP / dDI.

This mechanism may also contribute to the focal source hypothesis for AF, in which rapid regular regions activate too quickly for the remaining atrium, causing AF via fibrillatory conduction (Skanes et al. 1998, Waldo and Feld 2008).

Another option how to use MAPs can be examination of activations in the PVs and the vein of Marshall (VOM) during AF with congestive heart failure (CHF). Okuyama et al. (2003) revealed that AF in canine CHF is associated with independent focal activations in the PVs and the VOM, and with complex wave fronts within the PVs. Bipolar Hook electrodes were inserted into the right atrium and left atrium appendage for recording atrial electrograms and for burst pacing to induce atrial tachyarrhythmias. High-density mapping (1mm resolution) showed that the PV was activated by a focal wave front independent of left atrial (LA) activation in 22 AF episodes which were induced. These findings suggest that electrical and anatomical remodeling of the PVs and the VOM are important in the maintenance of AF in dogs. Mapping studies documented the presence of macro-reentry and fibrillatory conduction in the atria. These pro-arrhythmogenic effects are associated with extensive structural and electrical remodeling of atria particularly with atrial interstitial fibrosis and heterogeneous conduction delay.

Scherlag et al. (2008) proved that MAPs could be recorded also from the atrial appendages. This procedure then allows to detect substrates' effect such as neurotransmitters (norepinephrine or acetylcholine) using MAP upon the MS on PVs. This can be very helpful not only for studying the mechanisms underlying initiation of AF. It was demonstrated that acetylcholine injected into cardiac ganglionic plexi causes initiating of AF in PV in animal model (Patterson et al. 2006). Thus, it could be suggested that prevention of acetylcholine release from vagal nerve in order to avoid the vagal innervation of MS can also prevent initiation of AF.

Another practical use of MAPs is map-guided surgery of pulmonary vein isolation for AF. In some patients with persistent AF, disruption of the muscular connections between the PV and the LA may terminate the arrhythmia. The efficacy of mere epicardial PV isolation in the treatment of permanent chronic AF in comparison with the left atrial endocardial incisions was evaluated by Nitta et al. (2005). They hypothesized in their study that although current surgical procedures result in a high success rate for atrial fibrillation, they are not guided by electrophysiological findings in individual patients and thus might include unnecessary incisions in some patients or be inappropriate for other patients. Based on a study on 37 patients with permanent and 9 patients with paroxysmal AF who underwent surgical intervention for AF they concluded that intra-operative mapping is beneficial for the surgical treatment of AF and it also determining the optimal procedure with MAP detection of AF in these patients.

Vagal nerve suppression for atrial fibrillation was experimentally performed and recorded by MAPs. Suppression of AF by atropine was accompanied by improved sinoatrial (SA) and atrioventricular (AV) nodal function, increasing the ventricular heart rate observed during sinus rhythm and AF and eliminating the depressant actions of vagal nerve stimulation on SA and AV nodal function. Unlike atropine, mivacurium selectively antagonized the effects of vagal nerve and ganglionic plexus stimulation on atrial and pulmonary vein sleeve myocardium (shortening of action potential duration/refractoriness and increased atrial vulnerability) without altering sinus or AV nodal function under control conditions or during vagal nerve stimulation. The selective inhibition of parasympathetic nervous system 
effects in atrium versus sinus and AV nodes by mivacurium may represent a selective mechanism for the suppression of atrial fibrillation without altering SA and AV nodal function (Patterson et al. 2008).

Diagnostic and therapeutic evidence of premature atrial beats induced in MS of PVs and resulting in launch of AF was detected by 3D electroanatomic method of MAP. This method allowed to evaluate the changes in atrial response to vagal stimulation after PV ablation and also the effect of radiofrequency PV ablation itself. Moreover it proved that attenuated vagal response after ablation may also contribute to the suppression of AF.

\section{Conclusion}

Although a lot of evidences of the role of PVs myocardial sleeves in triggering AF were described this fact is from time to time neglected. The reason consists in the problem that ECG recording from a body surface can detect atrial fibrillation quite well but it cannot depict if AF was induced by PVs muscular sleeves or not. ECG videlicet cannot reveal such detailed local information like cellular depolarization and repolarization process of myocardial tissue. That is why MAP became an important instrument for measuring these processes depended directly on ionic currents. Computerized 3D electroanatomic mapping was implemented in order to help MAP to recognize focal activation in a PV more accurately. Intraoperative mapping not only identifies the patients who will be cured of AF by means of a simple procedure, such as PV isolation, but it also suggests where surgeons should place an additional ablation to cure AF (Nitta et al. 2005). Moreover the knowledge of pathological MAPs from PV myocardial sleeves can help us to confirm a diagnosis when finding the similar action potential morphology. MAP can be also used to evaluate the therapeutic efficiency of vagal nerves suppression, radiofrequency ablation or other treatment procedures in PVs myocardial sleeves as well as for post-treatment following up.

\section{Conflict of Interest}

There is no conflict of interest.

\section{Acknowledgements}

This study was supported by PRVOUK-P38/LF1/10 and SVV-2012-264504.

\section{References}

ALLESSIE MA, AUSMA J, SCHOTTEN U: Electrical, contractile and structural remodeling during atrial fibrillation. Cardiovasc Res 54: 230-246, 2002.

ARMOUR JA, MURPHY DA, YUAN B-X, MACDONALD S, HOPKINS DA: Gross and microscopic anatomy of the human intrinsic cardiac nervous system. Anat Rec 247: 289-298, 1997.

CHEN PS, CHOU CC, TAN AY, ZHOU S, FISHBEIN MC, HWANG C, KARAGUEUZIAN HS, LIN SF: The mechanisms of atrial fibrillation. J Cardiovasc Electrophysiol 17 (Suppl 3): S2-S7, 2006.

DE BAKKER JM, HO SY, HOCINI M: Basic and clinical electrophysiology of pulmonary vein ectopy. Cardiovasc Res 54: 287-294, 2002.

FRANZ MR: Method and theory of monophasic action potential recording. Prog Cardiovasc Dis 33: 347-368, 1991.

FYE WB: T. Lauder Brunton, 1844-1916. Clin Cardiol 12: 675-676, 1989.

GHERGHICEANU M, HINESCU ME, ANDREI F, MANDACHE E, MACARIE CE, FAUSSONE-PELLEGRINI MS, POPESCU LM: Interstitial Cajal-like cells (ICLC) in myocardial sleeves of human pulmonary veins. J Cell Mol Med 12: 1777-1781, 2008.

GO AS, HYLEK EM, PHILLIPS KA, CHANG Y, HENAULT LE, SELBY JV, SINGER DE: Prevalence of diagnosed atrial fibrillation in adults: national implications for rhythm management and stroke prevention: the AnTicoagulation and Risk factors In Atrial Fibrillation (ATRIA) Study. JAMA 285: 2370-2375, 2001.

HAÏSSAGUERRE M, JAÏS P, SHAH DC, TAKAHASHI A, HOCINI M, QUINIOU G, GARRIGUE S, LE MOUROUX A, LE MÉTAYER P, CLÉMENTY J: Spontaneous initiation of atrial fibrillation by ectopic beats originating in the pulmonary veins. N Engl J Med 339: 659-666, 1998.

HE B, SCHERLAG BJ, NAKAGAWA H, LAZZARA R, PO SS: The intrinsic autonomic nervous system in atrial fibrillation: a review. ISRN Cardiol 2012: Article ID 490674, 2012. 
HERTERVIG E, KONGSTAD O, LJUNGSTROM E, OLSSON B, YUAN S: Pulmonary vein potentials in patients with and without atrial fibrillation. Europace 10: 692-697, 2008.

HOFF HE, GEDDES LA: Cholinergic factor in atrial fibrillation. J Appl Physiol 8: 177-192, 1955.

JAÏS P, HAÏSSAGUERRE M, SHAH DC, CHOUAIRI S, CLÉMENTY J: Regional disparities of endocardial atrial activation in paroxysmal atrial fibrillation. Pacing Clin Electrophysiol 19: 1998-2003, 1996.

LEIRNER AA, CESTARI IA: Monophasic action potential. New uses for an old technique. Arq Bras Cardiol 72: 237242, 1999.

MOE GK: On the multiple wavelet hypothesis of atrial fibrillation. Arch Inter Pharm Ther 140: 183-188, 1962.

NARAYAN SM, KAZI D, KRUMMEN DE, RAPPEL WJ: Repolarization and activation restitution near human pulmonary veins and atrial fibrillation initiation: a mechanism for the initiation of atrial fibrillation by premature beats. J Am Coll Cardiol 52: 1222-1230, 2008.

NATHAN H, ELIAKIM M: The junction between the left atrium and the pulmonary veins. An anatomic study of human hearts. Circulation 34: 412-422, 1966.

NITTA T, ISHII Y, MIYAGI Y, OHMORI H, SAKAMOTO S, TANAKA S: Concurrent multiple left atrial focal activations with fibrillatory conduction and right atrial focal or reentrant activation as the mechanism in atrial fibrillation. J Thorac Cardiovasc Surg 127: 770-778, 2004.

NITTA T, OHMORI H, SAKAMOTO S, MIYAGI Y, KANNO S, SHIMIZU K: Map-guided surgery for atrial fibrillation. J Thorac Cardiovasc Surg 129: 291-299, 2005.

OKUYAMA Y, MIYAUCHI Y, PARK AM, HAMABE A, ZHOU S, HAYASHI H, MIYAUCHI M, OMICHI C, PAK HN, BRODSKY LA, MANDEL WJ, FISHBEIN MC, KARAGUEUZIAN HS, CHEN PS: High resolution mapping of the pulmonary vein and the vein of Marshall during induced atrial fibrillation and atrial tachycardia in a canine model of pacing-induced congestive heart failure. $J$ Am Coll Cardiol 42: 348-360, 2003.

OLSSON SB: Atrial fibrillation-where do we stand today? J Intern Med 250: 19-28, 2001.

PATTERSON E, LAZZARA R, SZABO B, LIU H, TANG D, LI YH, SCHERLAG BJ, PO SS: Sodium-calcium exchange initiated by the $\mathrm{Ca} 2+$ transient: an arrhythmia trigger within pulmonary veins. $J$ Am Coll Cardiol 47: 1196-1206, 2006.

PATTERSON E, LU Z, LIN J, SCHERLAG BJ, PO SS, COSCIA D, LAZZARA R: Antifibrillatory properties of mivacurium in a canine model of atrial fibrillation. J Cardiovasc Pharmacol 51: 293-303, 2008.

PAUZA DH, SKRIPKA V, PAUZIENE N, STROPUS R: Morphology, distribution, and variability of the epicardiac neural ganglionated subplexuses in the human heart. Anat Rec 259: 353-382, 2000.

PEREZ-LUGONES A, MCMAHON JT, RATLIFF NB, SALIBA WI, SCHWEIKERT RA, MARROUCHE NF, SAAD EB, NAVIA JL, MCCARTHY PM, TCHOU P, GILLINOV AM, NATALE A: Evidence of specialized conduction cells in human pulmonary veins of patients with atrial fibrillation. $J$ Cardiovasc Electrophysiol 14: 803-809, 2003.

SCHERF D: Studies on auricular tachycardia caused by aconitine administration. Proc Soc Exp Biol Med 64: 233-239, 1947.

SCHERF D, MORGENBESSER LJ, NIGHTINGALE EJ, SCHAEFFELER KT: Further studies on mechanism of auricular fibrillation. Proc Soc Exp Biol Med 73: 650-654, 1950.

SCHERLAG BJ, HOU YJ, LIN J, LU Z, ZACHARIAS S, DASARI T, NIU G, GHIAS M, PATTERSON E, JACKMAN WM, LAZZARA R, PO SS: An acute model for atrial fibrillation arising from a peripheral atrial site: evidence for primary and secondary triggers. J Cardiovasc Electrophysiol 19: 519-527, 2008.

SKANES AC, MANDAPATI R, BERENFELD O, DAVIDENKO JM, JALIFE J: Spatiotemporal periodicity during atrial fibrillation in the isolated sheep heart. Circulation 98: 1236-1248, 1998.

STEINER I: A hitherto unknown priority of Jan Ev. Purkyne - Myocardial sleeves of the pulmonary veins. Contribution to the pathogenesis of atrial fibrillation. CLC 144: 709-710, 2005.

STEINER I, HÁJKOVÁ P, KVASNICKA J, KHOLOVÁ I: Myocardial sleeves of pulmonary veins and atrial fibrillation: a postmortem histopathological study of 100 subjects. Virchows Arch 449: 88-95, 2006.

TAN AY, LI H, WACHSMANN-HOGIU S, CHEN LS, CHEN PS, FISHBEIN MC: Autonomic innervation and segmental muscular disconnections at the human pulmonary vein-atrial junction: implications for catheter ablation of atrial-pulmonary vein junction. J Am Coll Cardiol 48: 132-143, 2006. 
TSALIKAKIS DG, FOTIADIS DI, KOLETIS T, MICHALIS LK: Automated system for the analysis of heart monophasic action potentials. Comput Cardiol 30: 339-342, 2003.

WALDO AL, FELD GK: Inter-relationships of atrial fibrillation and atrial flutter mechanisms and clinical implications. J Am Coll Cardiol 51: 779-786, 2008.

YANG SG, KITTNAR O: New insights into application of cardiac monophasic action. Physiol Res 59: 645-650, 2010. ZEMLIN CW, MITREA BG, PERTSOV AM: Spontaneous onset of atrial fibrillation. Physica D 238: 969-975, 2009. 